\title{
Reflexões sobre a quarentena: uma estratégia de acolhimento de discentes em um grupo de mentoring
}

\author{
Reflections about quarantine: a strategy for undergraduate medical student reception in a mentoring group
}

\author{
Ana Carolina Rocha' (D) carolinapr.rocha@gmail.com \\ Isadora Falcão' (1) isadorafalcaofeitosa@gmail.com \\ João Guilherme Ávila de Lima' (1) joaoguilima@ufrn.edu.br \\ Jociele Moreira de Carvalho' (D) jociele29@hotmail.com \\ Maria Luisa de Oliveira Higino' (1) marialuisahigino17@gmail.com \\ \begin{tabular}{l|l} 
Rosiane Viana Zuza Diniz' ${ }^{1}$ & rosianevzdiniz@gmail.com
\end{tabular}
}

\begin{abstract}
RESUMO
Introdução: Notícias sobre a pandemia de Covid-19 chegam constantemente via meios de comunicação, e, rapidamente, há elevada sobrecarga de informações e inseguranças cotidianas. Para os estudantes de Medicina, essas mudanças podem ter efeito ainda mais significativo, pois já apresentam certo grau de adoecimento e queda da qualidade de vida pelo estresse acadêmico. Nessas situações, estratégias de reflexão, fala e escuta ativa podem ser úteis para a saúde mental.

Relato de experiência: Este relato apresenta, de forma descritiva, a análise reflexiva da percepção discente sobre facilidades e adversidades vivenciadas durante o distanciamento social proporcionado pela pandemia de Covid-19, bem como avalia de que modo a participação na mentoria interferiu nesse processo. Os estudantes responderam a um questionário de seis perguntas abertas sobre as experiências e a saúde mental deles. Esse questionário teve como objetivos refletir sobre o momento e compreendê-lo para melhorar a condução dos encontros do mentoring e minimizar um eventual impacto negativo na saúde mental dos discentes. Após a anuência dos participantes, por meio do Termo de Consentimento Livre e Esclarecido (TCLE) aprovado pelo Comitê de Ética em Pesquisa, as respostas foram analisadas em anonimato e coletivamente pelos próprios estudantes, de forma categorial temática, encontrando os núcleos de sentido a partir da leitura do corpus textual para identificação das unidades de análises.
\end{abstract}

Discussão: Houve 12 respondentes, o que representa uma taxa de resposta de 92,3\% dos estudantes aos quais foi enviado o questionário, no qual apontaram o impacto da pandemia na saúde mental, fizeram uma reflexão interior, indicaram os mecanismos adaptativos e apresentaram os aspectos da mentoria que a tornaram um ambiente seguro e de suporte.

Conclusão: A análise revelou que muitos discentes estão enfrentando dificuldades de adaptação, sobretudo quanto à saúde mental, como instabilidade emocional, revolta e frustração. Todavia, relataram-se positividade, desenvolvimento de hobbies e aumento do autoconhecimento e da comunicação com familiares. Apesar das limitações dos encontros remotos, não houve prejuízos de aproveitamento do programa e alcance de seus objetivos.

Palavras-chave: Tutoria; Educação Médica; Graduação; Saúde Mental; Covid-19.

\begin{abstract}
Introduction: The constant stream of news about the Covid-19 pandemic in the media has contributed to information overload and daily insecurities. For medical students, these changes can have an even more significant effect, as they already suffer a certain degree of illness and their quality of life is impaired by academic stress. In these situations, strategies for reflection, speech and active listening can be useful for mental health.

Experience report: This report presents, in a descriptive way, the reflective analysis of the student's perception of the facilities and adversities experienced during the social distancing measures enforced by the Covid-19 pandemic and how participation in mentoring interfered in this process. The students answered a questionnaire of six open-ended questions about their experiences and mental health, to reflect and better understand the current situation and thus enhance the mentoring program meetings, supporting and minimizing any negative impact on the students' mental health. After informed consent was agreed by the participants and approval given by the Ethics Committee, thematic content analysis of the answers was performed anonymously and collectively by the students themselves. The core meanings were sought from reading the textual corpus, thus identifying the units of analysis.
\end{abstract}

Discussion: There were twelve respondents, representing a response rate of $92.3 \%$ of the students to whom the questionnaire was sent, reporting the impact on their mental health, their inner reflections and adaptive mechanisms and mentoring as a safe environment of support.

Conclusion: The analysis revealed that many students are facing difficulties in adapting, especially regarding mental health, reporting issues such as emotional instability, revolt and frustration. However, positivity, development of hobbies, increased self-knowledge and communication with family members were reported. Despite the limitations presented by the remote meetings, there were no adverse effects of using the program and achieving its objectives.

Keywords: Mentoring; Medical Education; Undergraduate Training; Mental Health; COVID-19.

1 Universidade Federal do Rio Grande do Norte, Natal, Rio Grande do Norte, Brasil.

Editora: Lia Marcia Cruz da Silveira

Recebido em 02/03/21; Aceito em 27/04/21.

Avaliado pelo processo de double blind review. 


\section{INTRODUÇÃO}

Repentinamente, a pandemia decorrente do novo coronavírus (Covid-19) interrompeu não apenas as rotinas, como também as existências de muitas famílias pelo mundo todo'. Em um cenário em que as notícias e cobranças chegam constantemente via telecomunicação e mídia social, não é difícil que em pouco tempo haja elevada sobrecarga de informações e inseguranças quanto ao momento vivido. As atualizações nos números e as decisões estratégicas com relação à pandemia chegam rápido, e a população, como uma esponja, as absorve, determinando excessiva preocupação quanto à saúde e ao futuro após a pandemia².

Para os estudantes de Medicina, que frequentemente apresentam certo grau de adoecimento e queda da qualidade de vida devido ao estresse acadêmico, essas mudanças podem ter efeito ainda mais significativo ${ }^{3}$. A possibilidade de atuação como voluntário no enfrentamento da pandemia e a incerteza sobre seu futuro acadêmico figuram como preocupações adicionais para esses discentes no momento atual ${ }^{4}$.

A mentoria acadêmica consiste em um programa no qual um mentor orienta e estimula um grupo de alunos que trocam experiências entre si ao longo do curso de Medicina ${ }^{5}$. Em geral, o mentor é um docente do curso, e os encontros ocorrem quinzenalmente em horários que não comprometem as atividades acadêmicas obrigatórias ${ }^{6}$. Os alunos compartilham suas experiências e inseguranças dentro de um ambiente seguro. Esse acolhimento e essa troca de experiências mostramse especialmente importantes em momentos de instabilidade? ${ }^{7}$.

O curso de Medicina da Universidade Federal do Rio Grande do Norte (UFRN) iniciou o programa de orientação acadêmica no formato de mentoring em $2015^{8}$. Esse programa é composto por 25 grupos de mentoria no total (um mentor e até 22 mentorandos por grupo), sendo o mentor responsável pela forma como os encontros são realizados, adequando a metodologia e o tema abordado. Atualmente participam do programa 317 estudantes, representando $55,2 \%$ do total dos matriculados no curso. O grupo de mentores reúnese semestralmente para ajustes no desenvolvimento dos encontros para o semestre seguinte. Durante as reuniões, são compartilhadas as experiências que podem ser reproduzidas por outros grupos.

Este relato tem como objetivo descrever a percepção discente, os aspectos positivos, as fragilidades e o papel da mentoria, vivenciada durante o período de distanciamento social imposto pela pandemia de Covid-19, como estratégia norteadora para o desenvolvimento dos encontros em um grupo de mentoria do programa de mentoring do curso de Medicina da UFRN. Ademais, pretende valorizar, como parte visível de todo o processo relatado, uma construção autoetnográfica que ressalta o engajamento, a reflexão e os participantes ${ }^{9}$.

\section{RELATO DE EXPERIÊNCIA}

Este relato apresenta de forma descritiva a análise reflexiva da percepção discente sobre as facilidades e adversidades vivenciadas durante o período de distanciamento social imposto pela pandemia de Covid-19.

No início das atividades remotas do programa de mentoring, os estudantes foram convidados a responder a um questionário composto por seis perguntas abertas sobre suas experiências durante o período de distanciamento social (Quadro 1), pensando na repercussão provocada na saúde mental desse grupo e nos mecanismos encontrados para enfrentar tais dificuldades. O questionário foi elaborado por uma das autoras, a mentora do grupo respondente, e as respostas obtidas foram a base para a construção do relato de experiência. Nesse sentido, o questionário teve como objetivo compreender a percepção dos mentorados perante a quarentena imposta durante a pandemia de Covid-19.

Essa atividade teve como objetivos permitir a reflexão do estudante sobre o momento vivido e, a partir dessa compreensão, promover a condução dos encontros síncronos, com a perspectiva de fornecer apoio e minimizar o eventual impacto negativo do isolamento social na saúde mental desse grupo. Ao final do encontro, os mentorandos foram convidados a elaborar um relato de experiência sobre tais reflexões, tendo como base as respostas obtidas no questionário. Cinco mentorandos aceitaram o convite, e, com isso, realizaram-se reuniões para o planejamento das ações e para a elaboração do relato e análise anônima das respostas do questionário, além da discussão acerca dos possíveis temas centrais encontrados nas respostas do questionário. A anuência dos participantes

Quadro 1. Questões enviadas aos participantes do programa de mentoring para o início dos encontros durante a pandemia de Covid-19.

Como você tem vivenciado este período de quarentena?

Pense sobre ações e sentimentos que têm permeado o período de quarentena?

Que experiência você teve neste período de quarentena? O que você fez durante este período?

Gostaria de ter feito nesta quarentena......

A quarentena me trouxe uma grata (boa) surpresa! ......

A quarentena me trouxe uma surpresa não tão boa assim!.......

Fazer parte do mentoring neste período tem sido .... e porque...... 
foi obtida por meio do Termo de Consentimento Livre e Esclarecido (TCLE) aprovado pelo Comitê de Ética em Pesquisa da instituição: Certificado de Apresentação para Apreciação Ética (CAAE) n 38977020.2.0000.5292 e Parecer n 4.348.992.

As respostas foram analisadas de forma coletiva pela mentora e pelos cinco mentorados, preservando o anonimato dos respondentes. Promoveu-se a análise de conteúdo com categorização temática, buscando encontrar os núcleos de sentido a partir da leitura do corpus textual para identificação das unidades de análises, agrupadas em categorias e subcategorias a posteriori ${ }^{10,11}$.

Os discentes envolvidos no relato pertencem a um grupo de mentoria do programa de mentoring do curso de Medicina da UFRN. Esse programa é composto por 13 mentorandos, dos quais 12 (92,3\%) responderam ao questionário, que cursam do primeiro ao quarto ano (do primeiro ao oitavo semestre) do curso de Medicina (Gráfico 1). Dentre os respondentes, $50 \%$ eram homens.

Avaliaram-se por categorias os âmbitos citados pelos alunos nos relatos contidos em cada questionamento. Da análise de conteúdo, emergiram as seguintes categorias: "impacto na saúde mental", com as subcategorias "repercussões pessoais e profissionais" e "dificuldade na gerência do tempo"; "reflexão interior e mecanismos adaptativos", com as subcategorias "resgate de antigos hábitos e descoberta de novas habilidades" e "fortalecimento de vínculos familiares e de amizades"; e "a mentoria como ambiente seguro e de suporte", com as subcategorias "proximidade com a identidade profissional" e "fortalecimento do pertencimento pelas experiências compartilhadas" (Tabela 1).

\section{Impacto na saúde mental}

Dessa categoria emergiram a subcategoria "repercussões pessoais e profissionais", alcançada a partir do questionamento sobre como os participantes estavam vivenciando o período de isolamento e sobre quais surpresas negativas surgiram durante esse tempo. Quando perguntados sobre o que gostariam de ter feito durante a quarentena, os

Gráfico 1.Caracterização dos respondentes do questionário.

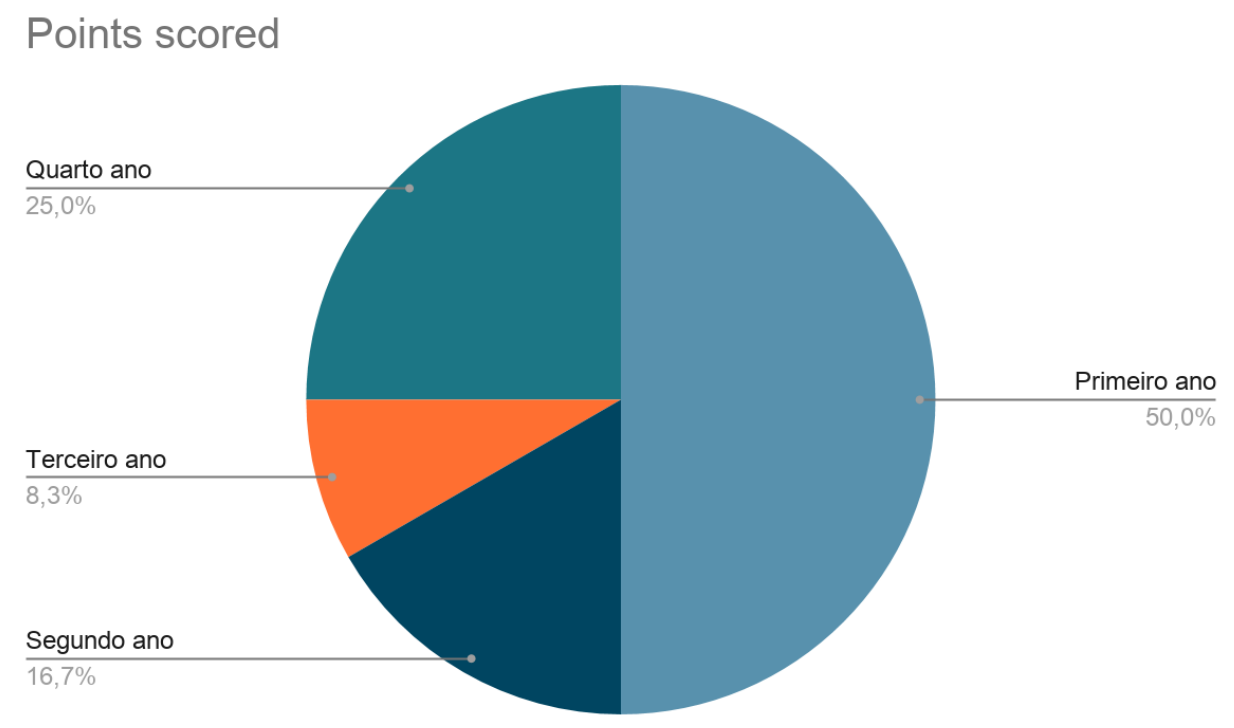

Tabela 1. Resultados obtidos em termos de categoria, subcategoria, unidades de análise e número/ frequência das unidades de análises.

\begin{tabular}{llc}
\hline \multicolumn{1}{c}{ Categoria } & \multicolumn{1}{c}{ Subcategoria } & Unidades de análise \\
\hline Impacto na saúde mental & Repercussões pessoais e profissionais & 19 \\
& Dificuldade na gerência do tempo & 3 \\
\hline $\begin{array}{l}\text { Reflexão interior e mecanismos } \\
\text { adaptativos }\end{array}$ & Resgate de antigos hábitos e descoberta de novas habilidades & 18 \\
\hline $\begin{array}{l}\text { A mentoria como ambiente seguro e } \\
\text { de suporte }\end{array}$ & Fortalecimento de vínculos familiares e de amizades & 7 \\
\hline
\end{tabular}


respondentes evidenciaram a "dificuldade na gerência do tempo", no decorrer do isolamento social.

\section{Reflexão interior e mecanismos adaptativos}

Referente a essa categoria, obtiveram-se respostas que foram agrupadas nas subcategorias "resgate de antigos hábitos e descoberta de novas habilidades" e "fortalecimento de vínculos familiares e de amizades", obtidas a partir do questionamento sobre as experiências e as boas surpresas que surgiram durante o período de distanciamento.

\section{A mentoria como ambiente seguro e de suporte}

O papel do mentoring caracterizou a terceira categoria. Quando questionados sobre a importância da sua participação nas atividades do programa mentoring, os respondentes apresentaram como significativas as experiências de "proximidade com a identidade profissional" e "fortalecimento do pertencimento pelas experiências compartilhadas".

De maneira geral, o decurso do distanciamento social afetou a saúde mental dos estudantes e trouxe preocupações pessoais e profissionais, as quais foram mencionadas 19 vezes pelos respondentes. "Dificuldade na gerência do tempo", citada três vezes, surgiu como um dos maiores motivos de preocupação e ansiedade dos participantes.

Diante da mudança de rotina e das novas pressões a que estavam submetidos, os alunos revelaram ter resgatado antigos hábitos, bem como novas habilidades e hobbies, e esse mecanismo adaptativo foi citado 18 vezes. Outra maneira de lidar com o período de isolamento mencionada pelos respondentes foi o "fortalecimento de vínculos familiares e de amizades", citado sete vezes.

Em meio ao desequilíbrio imposto pela quebra da rotina dos estudantes e pelas preocupações referentes à saúde mental e à formação profissional, o grupo do mentoring representou um ambiente seguro de suporte para os alunos, que identificaram no grupo o local de apoio nesse momento atípico, sendo mencionado diversas vezes pelos respondentes.

Os alunos descreveram que a participação no grupo do mentoring foi fundamental para que eles compartilhassem vivências e experiências e não se sentissem isolados, mesmo durante o tempo de distanciamento. A escuta do grupo e a orientação da mentora responsável permitiram que eles se sentissem acolhidos, compreendidos e respeitados, o que foi considerado uma vivência positiva para todo o grupo. Dessa forma, promoveu-se, por meio da escuta ativa e troca de aprendizados, um processo de ressignificação dos acontecimentos, sentidos e sentimentos com relação ao decurso do isolamento social, na medida em que novas percepções sobre a realidade foram formadas, transformadas e compartilhadas.
Em geral, os alunos atribuíram à participação nos encontros do mentoring um sentimento de pertencimento, referido por três respondentes, bem como a aproximação com a identidade profissional, mesmo que fisicamente distante do ambiente usual de aulas e práticas, que foi citada por cinco respondentes.

\section{DISCUSSÃO}

Este relato aponta para o relevante papel do mentor e da mentoria no curso de Medicina, corroborando o conceito apresentado por Botti et al. ${ }^{12}$, segundo o qual a função da mentoria deve avançar para além da orientação de aprendizagem pautada nos objetivos do curso, o que significa dar suporte ao educando para que ele alcance seus objetivos pessoais considerando a sua formação ${ }^{13}$. As vivências nas esferas acadêmica, familiar e social relatadas pelos mentorados e os eventos potencialmente adversos desencadeados pela pandemia de Covid-19 ressaltam a fundamental importância da interação mentor-mentorado estabelecida no período, tendo sido reconhecida como uma boa estratégia de enfrentamento para mitigar as implicações advindas da pandemia.

Nesse contexto, espera-se que o mentor tenha compreensão da prática profissional em sua essência e também estimule o desenvolvimento pessoal dos estudantes, bem como há a expectativa de que ele atue como guia, modelo e suporte ${ }^{12}$. Tais aspectos foram destacados pelos participantes nas falas apresentadas a seguir, nas quais se evidencia o ambiente seguro e de apoio, com resgate da identidade profissional.

Uma forma de enxergar que não estou sozinho [...]. Isso porque o diálogo é estimulado, as vivências são compartilhadas e os temas trazidos são pertinentes e motivadores (E10).

Permitiu compartilhar as experiências e me sentir acolhida (E11).

Às vezes, dá até para esquecer que faço Medicina. Nesse sentido, o mentoring permite uma quebra desse cenário, pois me coloca em contato com colegas de períodos mais avançados, que já estão se inserindo na rotina da profissão, assim como com um professor formado (E12).

Em geral, os alunos demonstraram majoritariamente emoções negativas sobre as vivências da quarentena, confirmando o impacto na saúde mental ocasionado pelo isolamento social e por suas repercussões ${ }^{2}$. Ademais, a incerteza com relação ao futuro e a angústia pela imprevisibilidade do momento vivido têm relação com a dinâmica massiva e a constante atualização de informações acerca da doença e de suas consequências ${ }^{2}$. Ainda, fatores ligados a questões acadêmicas, como a volta da rotina e o retorno das atividades, enquadram- 
se no tocante à grande preocupação dos estudantes do curso de Medicina com a formação profissional ${ }^{14}$.

No que concerne a essa percepção inicial, temos um panorama que pode ser bem sintetizado por falas como:

A ansiedade trouxe à tona alguns probleminhas internos com os quais sempre lutei, ansiedade, depressão, baixa autoestima [...] (E7).

Gostaria de ter estudado e lido mais e utilizado meu tempo livre disponível de forma mais organizada, responsável e produtiva (E5).

Logo, estabeleceu-se a necessidade de um ambiente de suporte que trabalhe as perspectivas vivenciadas por esses estudantes sob uma ótica bem estruturada. A perspectiva da mentoria consiste, portanto, em trazer o mentor como alguém que deve prover segurança emocional e suporte, de modo a não realizar julgamentos ou censuras, com o objetivo de apoiar o aprendiz de forma empática em suas dificuldades ${ }^{15}$.

Ademais, essa busca constante pelas atividades acadêmicas evidencia um traço perfeccionista característico dos estudantes dos cursos médicos, em que os alunos, apesar de estudarem e cumprirem com as suas obrigações, têm a sensação de que não fazem o suficiente ${ }^{16}$. Dessa forma, a participação em uma atividade de caráter optativo como a mentoria, além de ser uma estratégia de acolhimento, é uma oportunidade acadêmica de troca, mesmo em ambiente virtual, em que o mentorado aumenta sua autoeficácia, a satisfação com o trabalho e a produtividade; o mentor tem oportunidade de compartilhar sua experiência e sabedoria ${ }^{17}$, bem como ganha-se mutuamente um vínculo mais emocional e afetivo com o meio acadêmico e seus integrantes, o que é evidenciado nos relatos da seguinte forma:

Tem sido uma experiência importante, porque estou no primeiro período do curso e, com as aulas EaD, praticamente não tenho vivências mais diretas do cotidiano da minha futura profissão (E12).

Quanto às reflexões interiores e aos mecanismos adaptativos, foram relatados principalmente aspectos relacionados ao resgate de hábitos, ao desenvolvimento de novas habilidades e ao fortalecimento de vínculos. Com mais tempo livre na quarentena, os alunos puderam voltar o olhar para si mesmos e refletir sobre o mundo exterior, os acontecimentos e as pessoas que os rodeiam, como demonstrado na seguinte fala:

A quarentena me fez conhecer e me aproximar de muitas pessoas! Mesmo distantes, pude conversar com muita gente que eu havia perdido o contato na correria do dia a dia (E6).

Desse modo, o despertar dessa autoconsciência tem importância na prática médica, na medida em que propicia uma maximização no potencial da relação médico-paciente ${ }^{18}$, visto que torna o estudante mais preparado e aberto para lidar com o outro e com a vivência em grupo.

No resgate de hábitos no período de isolamento social, os estudantes relataram práticas de autocuidado, como a vivência de hobbies e atividades de lazer, tempo para descanso e rotinas de exercícios físicos. Essa é uma abordagem que se repete bastante e que pode ser ilustrada pelo depoimento de E5:

Tenho assistido filmes e séries, estudado, cozinhado, passado mais tempo com a minha família. (E5).

Os alunos têm dificuldade em priorizar ações de autocuidado em função da estrutura sobrecarregada do curso $^{19}$. Isso corrobora para que estejam mais expostos a fatores condicionantes e desencadeantes de adoecimento mental ${ }^{20}$. A mentoria é uma resposta institucional, bem como uma medida preventiva adotada pelas escolas médicas para superar tal adversidade ${ }^{20}$, e, por isso, merece ser estimulada, principalmente durante a pandemia.

Por fim, os aspectos positivos foram a aproximação com a família e o estreitamento de laços sociais. Nesse contexto, o apoio social promovido pela família ou mentoria é um dos mecanismos de enfrentamento do estresse. Devido ao grande grau de dedicação exigido pelo curso de Medicina, muitos alunos enfrentam grandes cargas de estresse, o que é capaz de diminuir a capacidade empática dos discentes. Sendo assim, o sentimento de empatia é essencial para a prática médica e afeta positivamente a qualidade de vida dos indivíduos ${ }^{21}$. Nesse sentido, a mentoria é vista pelos alunos como um ambiente seguro, como é possível observar em alguns relatos de alunos quando foram perguntados sobre como era fazer parte da mentoria:

Acolhedor, porque poder trocar experiências com colegas e com a professora me faz sentir parte de um grupo (E8).

Assim, é possível perceber que as relações sociais têm um papel importante no que tange ao enfrentamento dos desafios impostos pela pandemia de Covid-19 pelos estudantes do curso de Medicina. Ademais, ações da comunidade acadêmica e dos próprios estudantes que visem fortalecer esses vínculos são essenciais e devem ser incentivadas ${ }^{21}$.

\section{CONCLUSÃO}

Indiscutivelmente, a crise gerada pelo novo coronavírus acarretou transformações em diversos setores sociais e educacionais, levando à adequação das metodologias utilizadas. No relato apresentado, evidenciou-se o processo de adequação de metodologia para o programa de mentoring do curso de Medicina, uma vez que o questionário utilizado 
favoreceu a compreensão da realidade dos mentorados de maneira mais completa.

Nesse contexto, a análise das respostas dos discentes sobre seus sentimentos, suas vivências e atividades durante o período revelou que muitos enfrentam dificuldades de adaptação quanto à saúde mental, devido à alta frequência de relatos exibindo sentimentos negativos, instabilidade emocional e frustração.

Ademais, é importante salientar a existência de questões positivas na vivência da quarentena entre os estudantes, pois os relatos incluíam desenvolvimento de hobbies, autoconhecimento, comunicação com familiares, gratidão e respeito pela vida.

Dessa forma, constata-se que, apesar das limitações da transição entre os encontros presenciais e remotos, não houve prejuízo no aproveitamento do programa. Embora este relato apresente a experiência de apenas um grupo de mentoria, a relevância das percepções e reflexões obtidas possibilitou alcançar os objetivos da mentoria. Assim, tal relato pode permitir a ampliação da estratégia para outros grupos em nossa e/ou em outras instituições.

Essa experiência inicial foi considerada exitosa e deu origem a um projeto de pesquisa, ampliando a compreensão do distanciamento social para educandos e educadores de todo o curso de Medicina, bem como para os residentes em saúde. Os resultados vindouros podem permitir o fortalecimento e a ampliação das estratégias de suporte psicossocial para a formação qualificada de graduandos e residentes das profissões da saúde.

\section{CONTRIBUIÇÃO DOS AUTORES}

Ana Carolina Rocha, Isadora Falcão, João Guilherme Ávila de Lima, Jociele Moreira de Carvalho e Maria Luisa de Oliveira Higino participaram da análise e interpretação dos dados, e da escrita e aprovação da versão final do manuscrito. Rosiane Viana Zuza Diniz participou da organização, análise e interpretação dos dados, síntese, correção e revisão do texto, e da aprovação da versão final do manuscrito.

\section{CONFLITO DE INTERESSES}

Declaramos não haver conflito de interesses.

\section{FINANCIAMENTO}

Declaramos não haver financiamento.

\section{REFERÊNCIAS}

1. World Health Organization. Director-general's opening remarks at the media briefing on COVID-19. WHO; 2020 [acesso em 28 fev 2021]. Disponível em: https://www.who.int/director-general/speeches/detail/ who-director-general-s-opening-remarks-at-the-media-briefing-oncovid-19---11-may-2020.

2. Schmidt B, Crepaldi MA, Bolze SDA, Neiva-Silva L, Demenech LM. Saúde mental e intervenções psicológicas diante da pandemia do novo coronavírus (Covid-19). Estud Psicol. 2020;37:1-13.

3. Moreira SNT, Vasconcellos RLSS, Heath N. Stress in medical education: how to face this reality? Rev Bras Educ Med. 2015;39(4):558-64.

4. O'Byrne L. Medical students and COVID-19: the need for pandemic preparedness. J Med Ethics. 2020;46(9):623-6.

5. Alpes $M$, Wolf A. Tutoria acadêmica ("mentoring"): relato de experiência de um tutorado a tutor. Extensão em Foco. 2018;(16):90-8.

6. Hyppolito MA, Elias LLK. O Programa de Educação Tutorial na Faculdade de Medicina de Ribeirão Preto, Universidade de São Paulo. Medicina (Ribeirão Preto). 2016;49(4):367-8.

7. Martins AF, Bellodi PL. Mentoring: uma vivência de humanização e desenvolvimento no curso médico. Interface Comun Saúde Educ. 2016;20(58):715-26.

8. Moreira SNT, Albuquerque ICS, Pinto Junior FEL, Gomes AHB. Programa de Mentoria do curso de Medicina da Universidade Federal do Rio Grande do Norte: atividades integrativas em foco. Rev Bras Educ Med. 2020;44(4):e169.

9. Jones $\mathrm{SH}$, Adams TE, Ellis C, Oliveira MAO, Jaramillo NJ. Handbook of autoethnography. Walnut Creek: Left Coast Press; 2013.

10. Bardin L. Análise de conteúdo. Lisboa: Edições 70;1988.

11. Minayo MCS. O desafio do conhecimento: pesquisa qualitativa em saúde. Cien Saude Colet. 2007;2(4):1087-8.

12. Botti SHO, Rego S. Preceptor, supervisor, tutor e mentor: quais são seus papéis? Rev Bras Educ Med. 2008;32(3):363-73.

13. Martins AF, Bellodi PL. Mentoring in medical students: a humane and developmental experience. Interface (Botucatu). 2016;20(58):715-26.

14. Moromizato MS, Ferreira DBB, de Souza LSM, Leite RF, Macedo FN Pimentel D. O uso de internet e redes sociais e a relação com indícios de ansiedade e depressão em estudantes de Medicina. Rev Bras Educ Med. 2017;41(4):497-504

15. Chaves LJ, Gonçalves ECQ, Ladeira LR, Ribeiro MS, Costa MB, Ramos AAM A tutoria como estratégia educacional no ensino médico. Rev Bras Educ Med. 2014;38(4):532-41,

16. Henning K, Ey S, Shaw D. Perfectionism, the impostor phenomenon and psychological adjustment in medical, dental, nursing and pharmacy students. Med Educ. 1998;32(5):456-64.

17. Burgess A, Diggele CV, Mellis C. Mentorship in the health professions: a review. Clin Teach. 2018;15:197-202.

18. Ouliaris $C$. The importance of self-awareness: musings of a medical student. Australas Psychiatry. 2019;27(3):267-9.

19. Ayala EE, Omorodion AM, Nmecha D, Winseman JS, Mason HRC What do medical students do for self-care? A student-centered approach to well-being. Teach Learn Med. 2017;29(3):237-46. doi: 10.1080/10401334.2016.1271334.

20. Conceição LS, Batista CB, Dâmaso JGB, Pereira BS, Carniele RC, Pereira GS. Saúde mental dos estudantes de medicina brasileiros: uma revisão sistemática da literatura. Avaliação: Revista da Avaliação da Educação Superior. 2019;24(3):785-802.

21. Park KH, Kim D, Kim SK, Yi YH, Jeong JH, Chae J, et al. The relationships between empathy, stress and social support among medical students. Int J Med Educ. 2015;6:103-8. 